\title{
Traduire
}

Une autre perspective sur r tr traduction

Revue française de la traduction

$219 \mid 2008$

Le temps de la réflexion

\section{Compte rendu d'ouvrage : Guide anglais-français de la traduction de René Meertens}

\section{Aurélie Barbe}

\section{(2) OpenEdition}

1 Journals

Édition électronique

URL : http://journals.openedition.org/traduire/881

DOI : $10.4000 /$ traduire.881

ISSN : 2272-9992

Éditeur

Société française des traducteurs

Édition imprimée

Date de publication : 15 décembre 2008

Pagination : 84-87

ISSN : 0395-773X

Référence électronique

Aurélie Barbe, "Compte rendu d'ouvrage : Guide anglais-français de la traduction de René Meertens »,

Traduire [En ligne], 219 | 2008, mis en ligne le 15 décembre 2008, consulté le 23 septembre 2020. URL http://journals.openedition.org/traduire/881 ; DOI : https://doi.org/10.4000/traduire.881 


\title{
Compte rendu d'ouvrage : Guide anglais-français de la traduction de René Meertens
}

\author{
AURÉlie BARBE
}

Le Guide anglais-français de la traduction de René Meertens, dont la $4 \mathrm{e}$ édition est parue en juin 2008, est devenu un ouvrage incontournable pour tous les traducteurs anglais $>$ français. Il s'agit là d'un complément indispensable aux dictionnaires bilingues généraux. Précisons que cet ouvrage, comme l'indique son titre, s'est, jusqu'à présent, uniquement intéressé au sens de traduction de l'anglais vers le français.

Ce dictionnaire traductif est le fruit de 30 années de travail au sein de grandes organisations internationales. Revenons sur le remarquable parcours de René Meertens, son auteur. René Meertens a commencé sa carrière de traducteur à l'ONU (New York) où il a exercé pendant deux ans. Il a ensuite travaillé pendant onze ans à la Commission européenne à Bruxelles dans le groupe Affaires sociales et transports puis dans le groupe Affaires économiques et financières. En 1991, le traducteur s'est vu offrir un poste au Bureau de l'Europe de l'OMS à Copenhague où il passe actuellement 6 mois par an. Depuis 1999, année de parution de l'ouvrage, René Meertens travaille également pour l'ONU en qualité de réviseur temporaire. Il a travaillé aux bureaux de New York, de Vienne et de Genève. Il passe actuellement deux mois par an dans cette dernière ville et consacre le reste de l'année à l'enrichissement de son ouvrage.

Le Guide anglais-français de la traduction regroupe aussi bien des termes généralistes susceptibles de poser des problèmes de traduction dans certains contextes (business, corporate, community, to ensure, to 
include par exemple) que des termes spécialisés (entre autres : informatique, économie/finance, droit, sciences, Union européenne), des acronymes et des termes propres à la culture anglo-saxonne souvent absents des dictionnaires bilingues (xanadu, weathering, BA (British Airways), TD (Teachtaí Dála), DWI (driving while intoxicated), $N G$ (newsgroup)).

Chaque entrée présente plusieurs significations du mot et des expressions contenant le terme en question. Les nombreuses traductions proposées apparaissent de façon ordonnée et sont illustrées par des exemples. On remarquera que des cooccurrents sont très souvent suggérés. Le traducteur ou l'étudiant en traduction pourra ainsi choisir la traduction la plus appropriée.

Prenons par exemple un terme dont la traduction varie énormément en fonction du contexte et du domaine concerné : le substantif scope. Le Guide propose les traductions suivantes :

1. Champ/domaine couvert/d'application, champ d'observation/ d'étude, questions étudiées/abordées, objet

2. Champ/rayon d'action, domaine d'activité

3. Domaine de compétence, ressort

4. Éventail, gamme, spectre

5. Ampleur [d'un problème], étendue [d'une obligation], objet [d'une réunion], portée, délimitation, contenu, définition, importance, envergure, dimension, extension, cadre, contours, limites ; périmètre ; paramètres

6. Latitude, liberté d'action, marge (de manœuvre/d'appréciation)

7. Possibilité(s) (d'action), perspectives, chances, place, potentiel, moyens

R. Meertens nous présente en plus la traduction idiomatique de quelques expressions contenant scope :

To fall within the scope of : entrer dans le champ d'application de, relever de, ressortir à ; [action illicite] tomber sous le coup de To fall 
outside the scope of : sortir du champ d'application de, sortir du cadre de, ne pas être du ressort de

There is scope for : il est possible de

Ainsi que des exemples d'emploi :

The scope of the report: les questions abordées dans le rapport, l'objet du rapport

Aesthetic questions are beyond the scope of this report : les questions esthétiques ne sont pas abordées dans ce rapport

The minister wanted to broaden the scope of the study: le ministre voulait élargir le champ de l'étude

This rule has truly universal scope : cette règle a une portée véritablement universelle

Issues beyond the scope of the report: questions qui débordent du cadre du rapport

There is no scope for tax cuts : aucune réduction d'impôts n'est possible There remains plenty of scope for an enquiring mind: un esprit curieux peut encore largement s'exprimer

La nouvelle édition, parue en juin 2008, compte 543 pages, soit plus de $10 \%$ de pages supplémentaires par rapport à l'édition précédente publiée en 2004. Cette augmentation provient de l'enrichissement d'articles déjà existants et de la création de nouveaux articles. Parmi ces derniers, figurent, entre autres, junket (voyage tous frais payés), le substantif juvenile (mineur), Kathmandu (Katmandou), kerning (crénage, terme informatique), keylogger (enregistreur de frappe, terme informatique), kiss of death (cadeau empoisonné ; échec assuré, terme familier), le substantif knock-off (copie, produit d'imitation) ou encore Kremlin watcher (kremlinologue).

Pour conclure, cet ouvrage fait gagner un temps précieux en recherche de synonymes, de termes spécialisés et culturels, d'abréviations et de 
collocations, et a assurément une place dans tous les bureaux des traducteurs professionnels. Rappelons toutefois que ce guide ne prétend pas à l'exhaustivité et que s'il contient de nombreux termes spécialisés, il s'agit avant tout d'un ouvrage de traduction généraliste (les termes très pointus n'y figurent pas). L'ouvrage n'est actuellement disponible que sur papier, mais étant donné l'utilité du Guide anglais-français de la traduction pour le quotidien des traducteurs, une version électronique serait la bienvenue.

Les lecteurs pourront consulter un extrait du Guide anglais-français de la traduction sur le site Internet de l'auteur à l'adresse suivante :

http://www.renemeertens.eu/files/Apercu.pdf

Notons que René Meertens est aussi l'auteur du Dictionnaire anglaisfrançais français-anglais de la santé et du médical. Ce dictionnaire bilingue de 284 pages est également publié par les Éditions Chiron.

Guide anglais-français de la traduction

René Meertens

Éditions Chiron

ISBN : 978-2702712467

$30 €$

543 pages, $17,5 \times 24 \mathrm{~cm}$ 